\section{From swimming to walking with a salamander robot driven by a spinal cord model}

Auke Jan Ijspeert ${ }^{1}$ \& Alessandro Crespi $^{1}$ \& Dimitri Ryczko ${ }^{2,3}$ \& Jean-Marie Cabelguen ${ }^{2,3}$

${ }^{1}$ School of Computer and Communication

Sciences, Ecole Polytechnique Fédérale de

Lausanne (EPFL), Station 14, CH-1015

Lausanne, Switzerland,

${ }^{2}$ INSERM, U 862, Bordeaux, F-33077, France,

${ }^{3}$ University Bordeaux 2, Bordeaux, F33077, France.

The transition from aquatic to terrestrial locomotion was a key development in vertebrate evolution. We present a spinal cord model and its implementation in an amphibious salamander robot that demonstrates how a primitive neural circuit for swimming can be extended by phylogenetically more recent limb oscillatory centers to explain the ability of salamanders to switch between swimming and walking. The model suggests neural mechanisms for modulation of velocity, direction, and type of gait that are relevant for all tetrapods. It predicts that limb oscillatory centers have lower intrinsic frequencies than body oscillatory centers, and we present biological data supporting this.

The salamander, an amphibian, is regarded as the tetrapod most closely resembling the first terrestrial vertebrates and represents therefore a key animal from which the evolutionary changes from aquatic to terrestrial locomotion can be inferred $(1,2)$. It is capable of rapidly switching between two locomotion modes: swimming and walking (3-5). The swimming mode is similar to that of the lamprey, a primitive fish, with fast axial undulations being propagated as traveling waves from head to tail, while the limbs are folded backwards. On firm ground, the salamander switches to a slower stepping gait, in which diagonally opposed limbs are moved together while the body makes S-shaped standing waves with nodes at the girdles (3-6).

Using the salamander as an animal model, we address three fundamental issues related to vertebrate locomotion: (i) the modifications undergone by the spinal locomotor circuits during the evolutionary transition from aquatic to terrestrial locomotion, (ii) the mechanisms necessary for coordination of limb and axial movements, and (iii) the

A

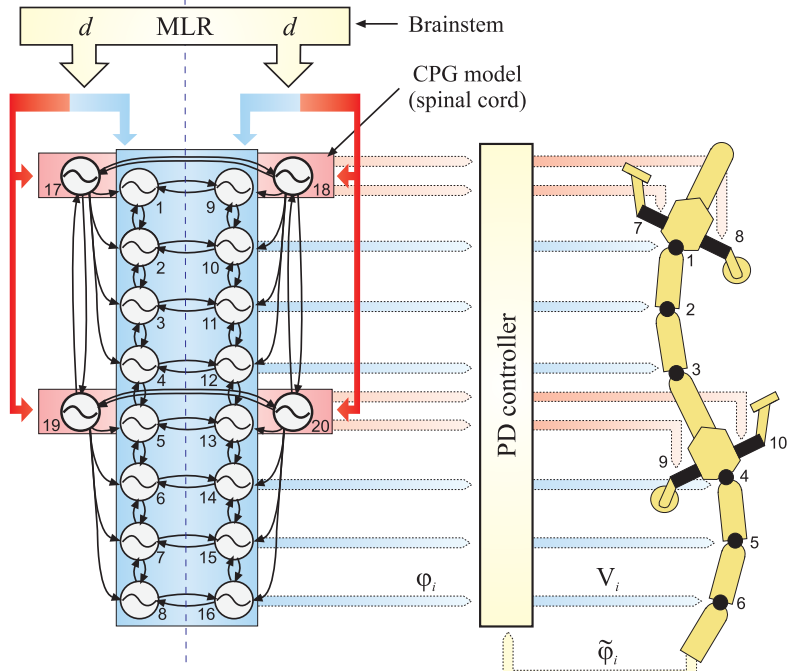

B

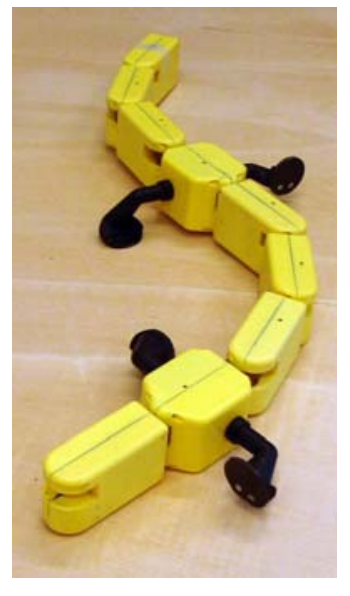

Fig. 1. Configuration of the CPG model (A) and salamander robot (B). The robot is driven by 10 DC motors, which actuate 6 hinge joints for the spine (black disks in the schematic view of the robot), and 4 rotational joints for the limbs (black cylinders). The CPG is composed of a body CPG — a double chain of 16 oscillators with nearest neighbor coupling for driving the spine motors — and a limb CPG — 4 oscillators for driving the limb motors. The outputs of the oscillators are used to determine the setpoints $\varphi_{i}$ (desired angles) provided to Proportional-Derivative (PD) feedback controllers that control the motor torques (through their voltage $V_{i}$ ) given the actual angles $\tilde{\varphi}_{i}$. The CPG model receives left and right drive signals $d$ from the MLR region in the brain stem. The velocity, direction and type of gait exhibited by the robot can be adjusted by modifying these two signals.

mechanisms that underlie gait transitions induced by simple electrical stimulation of the brain stem. We address these questions with the help of a numerical model of the salamander's spinal cord that we implement and test on a salamander-like robot capable of swimming and walking. Consequently, this study is also a demonstration of how robots can be used to test biological models, and in return, how biology can help in designing robot locomotion controllers.

As in other vertebrate animals, salamander gaits are generated by a central pattern generator (CPG) $(7,8)$. As in the lamprey $(9$, $10)$ and in the Xenopus embryo $(11,12)$, the CPG for axial motion - the body $C P G$ - is distributed along the entire length of the spinal cord. It forms a double chain of oscillatory centers (groups of neurons that exhibit rhythmic activity) located on both sides of the spinal cord, and generates traveling waves corresponding to fictive swimming when activated by $\mathrm{N}$-methylDaspartate bath application in isolated spinal cord preparations (7). The neural centers for the movements of the limbs —-forming the limb $\mathrm{CPG}$ - are located in the cervical segments for the forelimbs and in the thoraco- lumbar segments for the hindlimbs $(13,14)$. Locomotion can be induced by simple electrical stimulation of the mesencephalic locomotor region (MLR) located in the midbrain (15). Low levels of stimulation induce the slow walking gait and, at some threshold, higher stimulation induces a rapid switch to the faster swimming mode. In both modes, the frequency of motion is proportional to the stimulation strength. Gait transitions by MLR stimulation have been observed in all classes of vertebrates and appear to be a common property of vertebrate locomotor control (16).

Although these data show the general organization of the locomotor CPG, they do not explain how the different oscillatory centers are coupled together and how they are driven by command signals for gait generation and modulation. We have developed a numerical model of the salamander CPG to explore these questions, which are relevant to all tetrapods. Previous numerical models (1720) have provided insights into possible mechanisms for gait transition, but failed to explain the MLR stimulation experiment described above (15), and the observation that 
swimming frequencies are systematically higher than walking frequencies.

Our model is based on four main hypotheses. Hypothesis 1: The body CPG is like that of the lamprey and spontaneously produces traveling waves when activated with a tonic drive. The limb CPG, when activated, forces the whole CPG into the walking mode, as previously proposed in (1). Hypothesis 2: The strengths of the couplings from limb to body oscillators are stronger than those from body to body oscillators and from body to limb oscillators. This allows the limb CPG to "override" the natural tendency of the body CPG to produce traveling waves and force it to produce standing waves. Hypothesis 3 : Limb oscillators can not oscillate at high frequencies, that is, they saturate and stop oscillating at high levels of drive. This provides a mechanism for automatically switching between walking and swimming when the drive is varied (15), and explains why swimming frequencies are systematically higher than walking frequencies $(3,5)$. Hypothesis 4: For the same drive, limb oscillators have lower intrinsic frequencies than the body oscillators. This explains the rapid increase of frequency during the switch from walking to swimming and the gap between walking and swimming frequency ranges $(3,5)$.

The CPG model is composed of a body CPG and a limb CPG implemented as a system of coupled nonlinear oscillators (Fig. 1A). Similar to lamprey models (21), the bursting properties of an oscillatory center the oscillations between bursts of motoneuron activity and periods of rest - are modeled by means of a phase oscillator with controlled amplitude:

$$
\begin{aligned}
& \dot{\theta}_{i}=2 \pi v_{i}+\sum_{j} r_{j} w_{i j} \sin \left(\theta_{j}-\theta_{i}-\phi_{i j}\right) \\
& \ddot{r}_{i}=a_{i}\left(\frac{a_{i}}{4}\left(R_{i}-r_{i}\right)-\dot{r}_{i}\right) \\
& x_{i}=r_{i}\left(1+\cos \left(\theta_{i}\right)\right)
\end{aligned}
$$

Where $\theta_{i}$ and $r_{i}$ are the state variables representing the phase and the amplitude of oscillator $i, v_{i}$ and $R_{i}$ determine its intrinsic frequency and amplitude, and $a_{i}$ is a positive constant. Couplings between oscillators are defined by the weights $w_{i j}$ and phase biases $\phi_{i j}$. A positive oscillatory signal $x_{i}$ represents the burst produced by the center.

In the lamprey and the salamander, the amplitude and frequency of bursts depend on the amount of stimulation $(15,22)$. Typically, when an increasing drive is applied, three phases can be distinguished: (i) a sub-

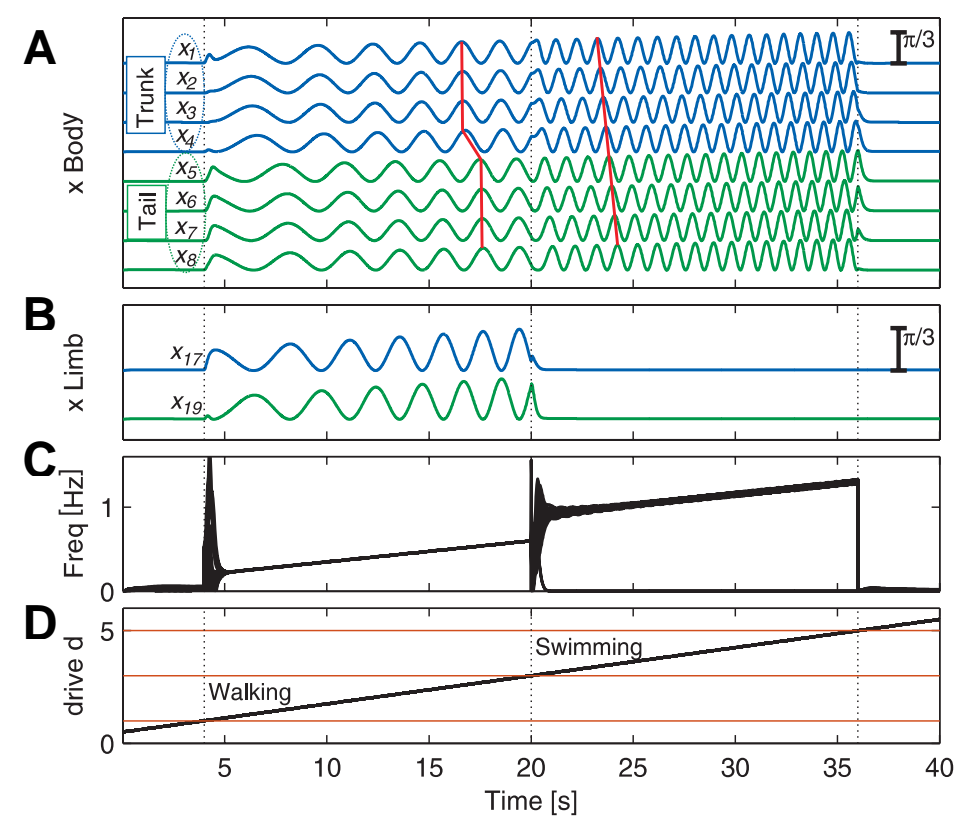

Fig. 2. Switching from walking to swimming; activity of the CPG model when the drive signal is progressively increased. (A) $x_{i}$ signals from the left body CPG oscillators (oscillators on the right side are exactly in anti-phase). The numbering corresponds to that of Fig. 1A. Units are in radians (scale bar on the top right). The red lines illustrate the transition from standing waves (with synchrony in the trunk, synchrony in the tail, and an anti-phase relation between the two, $4 \mathrm{~s}<\mathrm{t}<20 \mathrm{~s}$ ) to traveling waves (20s $<\mathrm{t}<36 \mathrm{~s}$ ). (B) $x_{i}$ signals from the left limb CPG oscillators. Ipsilateral fore- and hindlimbs are in anti-phase.

(C) Instantaneous frequencies measured as $\frac{\dot{\theta}_{i}}{2 \pi}$ in cycles/s. The variations in the instantaneous frequencies among individual oscillators at times $\mathrm{t}=4 \mathrm{~s}$ and $\mathrm{t}=20 \mathrm{~s}$ correspond to brief accelerations and decelerations before re-synchronization. (D) Linear increase of the drive $d$ applied to all oscillators. The horizontal red lines correspond to the lower $\left(d_{\text {low }}^{\text {limb }}=d_{\text {low }}^{\text {body }}=1\right)$ and upper $\left(d_{\text {high }}^{\text {limb }}=3, d_{\text {high }}^{\text {body }}=5\right)$ oscillation thresholds for limb and body oscillators in arbitrary drive units. Movie S2 shows a similar switch from walking to swimming in the robot.

threshold phase without bursts, (ii) an oscillating phase where the frequency and amplitude of bursts increase with the drive, and (iii) a saturation phase where centers stop oscillating. We replicate this effect by introducing a piece-wise linear saturation function which similarly modulates the intrinsic frequency and amplitude $v_{i}$ and $R_{i}$ according to a drive signal $d_{i}$ between a lower oscillation threshold $d_{\text {low }}$ and an upper one $d_{\text {high }}$. Limb and body oscillators are provided with different saturation functions, with the limb oscillators systematically oscillating at lower frequencies than body oscillators for the same drive (hypothesis 4) and saturating at a lower threshold $d_{\text {high }}$ (hypothesis 3). Except for turning, all oscillators receive the same drive $d$.

The coupling parameters $w_{i j}$ and $\phi_{i j}$ are set such that the body CPG produces traveling waves (hypothesis 1) and the limb CPG produces the salamander stepping. There are unidirectional couplings from limb oscillators to body oscillators (Fig. 1A) whose strengths are larger than those within the body CPG (hypothesis 2). More details and parameters are provided in the Supporting Online Material (23).

Robots are increasingly used as tools to test hypotheses concerning biological systems (24). Here, we test the spinal cord model on a salamander robot whose purpose is three-fold: (i) to show that our CPG model can generate forward motion with variable speed and heading (i.e., aspects that need a "body" for validation and cannot be studied at a neuronal level alone), (ii) to qualitatively compare the gaits generated to those of the real salamander, and (iii) to show that the concept of CPGs can lead to robust locomotion control for robots with multiple articulated joints.

The $85 \mathrm{~cm}$ long robot is designed to approximately match the kinematic structure 
A

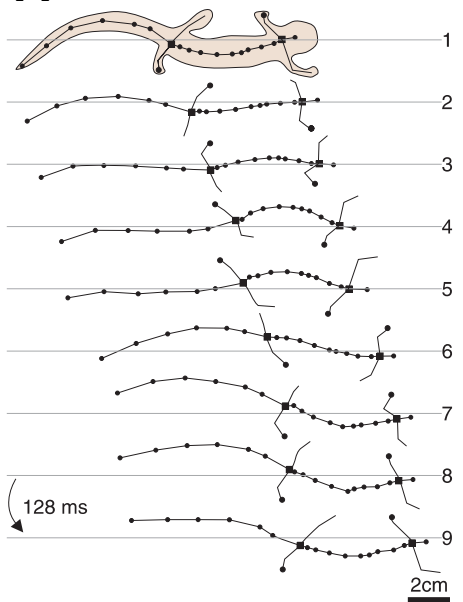

B

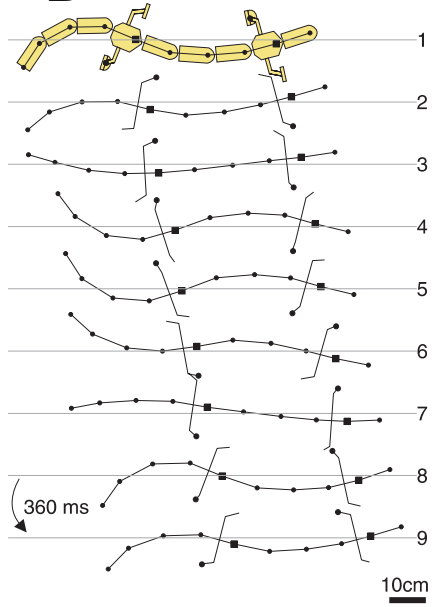

C

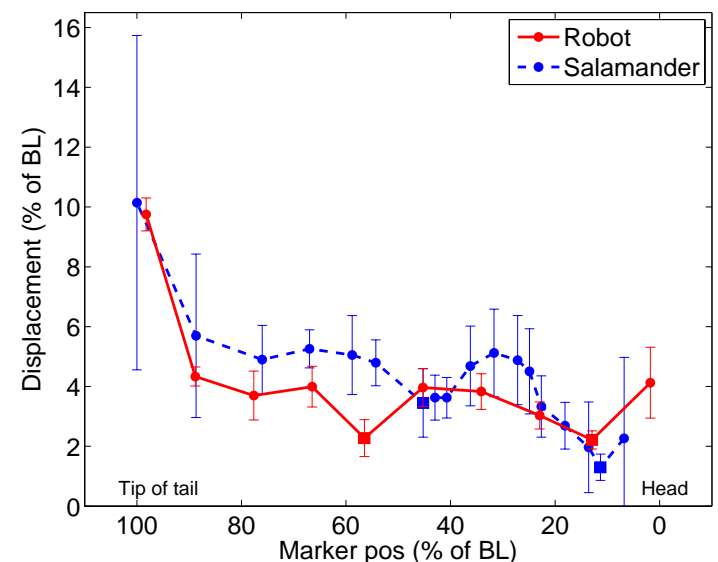

Fig. 3. Walking gait. (A) Successive midline profiles reconstructed from digitized video fields by means of 18 marker points (black dots) during a complete stepping cycle of one individual salamander (velocity $=0.06 \mathrm{~m} / \mathrm{s}=0.34 \mathrm{BL} / \mathrm{s}$ ). BL stands for body length. Squares indicate girdles. A dot at the extremity of a limb indicates the estimated foot contact with the ground. The horizontal lines show the overall direction of forward travel. (B) Same measurement with 10 markers on the robot (drive=2.0, velocity=0.06m/s=0.07BL/s). $(\mathrm{C})$ Envelopes corresponding to the maximal lateral displacements in the salamander and the robot. The data points and error bars correspond to the averages and standard deviations of 5 sequences at various velocities for the salamander and 25 sequences for the robot (23). See also the movies S1 and S2.
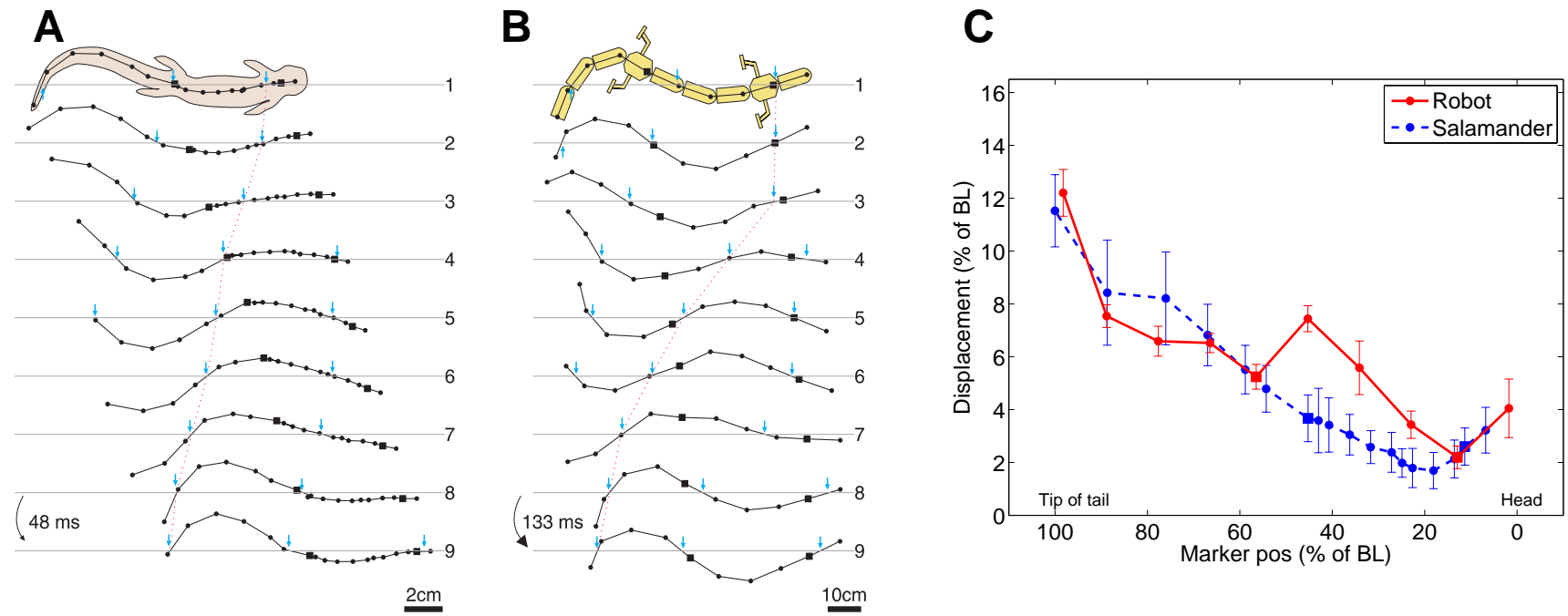

Fig. 4. Swimming mode. (A) Successive midline profiles during a complete swimming cycle of one individual salamander (velocity $=0.17 \mathrm{~m} / \mathrm{s}=0.89 \mathrm{BL} / \mathrm{s}$ ). Same representation as in Fig 3A. Arrows indicate the points of minimal lateral displacement from the overall direction of forward travel (horizontal lines). Note the traveling wave in the body undulation. (B) Undulations in the robot (drive $=4.0$, velocity $=0.11 \mathrm{~m} / \mathrm{s}=0.13 \mathrm{BL} / \mathrm{s}$ ). (C) Envelopes corresponding to the maximal lateral displacements. The data points and error bars correspond to the averages and standard deviations of 6 sequences at various velocities for the salamander and 25 sequences for the robot (23). See also the movies S1 and S2.

of salamanders (Fig. 1B). The robot can move its four limbs as well as produce lateral undulations of the spine with six actuated hinge joints. Unlike the real animal, limbs perform continuous rotation. The rotation replicates the rotational thrust that salamander legs apply to the ground while in stance phase, and allows the alternation between swing and stance. Setpoints for the motor controllers are based on the difference between the $x_{i}$ signals from the left and right body oscillators for the spine motors, and on the phases $\theta_{i}$ of the limb oscillators for the limb motors. See (23) for additional design information.

The CPG model produces swimming and walking patterns that are consistent with those of the real salamander. As observed in MLR stimulation experiments (15), the model produces an abrupt transition between gaits simply by varying the drive (Fig. 2). During walking (i.e., at low drive), the strong couplings from limb to body oscillators force the body CPG to oscillate at a low frequency with an S-shaped standing wave as in the electromyogram (EMG) recordings (5). The frequency and amplitude of oscillations increase proportionally with the drive. At $\mathrm{t}=20 \mathrm{~s}$, the limb oscillators saturate, and this 
induces a rapid gait transition to the higher frequency swimming mode. Traveling waves for swimming are released, also like in the EMG recordings (5). These traveling waves increase in amplitude and frequency as the drive is further increased, until the body oscillators reach their upper oscillation threshold and stop oscillating.

These numerical results agree with detailed kinematic analyses of the gait transitions which found that traveling waves in the body axis are not observed simultaneously with limb movements (4). Another important similarity with the MLR stimulation experiments and with recordings in tadpoles (12) is the step increase of frequencies during the transition from walking to swimming. In the model, the limb oscillators slow down the rhythms during walking, and once silent, rapidly release faster swimming rhythms due to the higher intrinsic frequencies of the body oscillators. This can also explain why salamander walking and swimming frequencies do not overlap $(3,5)$, but have distinct ranges with a gap between them (e.g., walking from 0.6 to $1.2 \mathrm{~Hz}$, swimming from 1.6 to $2.9 \mathrm{~Hz}$ in the salamander Pleurodeles waltlii). In our model, walking and swimming frequencies range respectively from 0.2 to 0.6 $\mathrm{Hz}$ and from 0.9 to $1.3 \mathrm{~Hz}$. The intrinsic frequencies of the model have been reduced compared to those of the real salamander to fit within the torque limits of the robot motors. Although Fig. 2 shows an example with a simple linear increase of the drive, the model can readily deal with abruptly and continuously varied drives (as likely occurs in a freely behaving animal) and modulate the velocity and type of gait accordingly (23).

In addition to similarities in neural patterns, the gaits produced by the robot are similar to those of a real salamander. In the walking gait (Fig. 3), the body makes an S-shaped standing wave with nodes at the girdles. The envelopes of lateral displacements compared to the direction of motion (Fig. 3C) are qualitatively similar for the robot and the salamander, with minimal displacements close to the girdles (note that the hindlimb girdle is located closer to the tail for the robot). The axial undulations resemble that of the salamander with two exceptions: The tail of the robot is bent over its whole length whereas the tip of salamander's tail tends to remain straight, and the head of the robot makes more lateral displacements because it lacks joints in the neck. The body-limb coordination in both the robot and the real salamander optimizes stride length (23). Increasing the drive leads to an augmentation of the speed of walking, due to the higher frequency and amplitude of oscillations. The walking velocities obtained range from 0.03 to $0.09 \mathrm{~m} / \mathrm{s}$ (0.04 to 0.11 body lengths/s). In relative terms, the robot is slower than a $P$. waltlii, which walks at velocities in the range of 0.1 to 0.4 body lengths/s. The difference can be explained by the lower frequencies used in the robot.

The swimming mode of the robot is also consistent with that of a real salamander (Fig. 4). The traveling wave of body undulation allows the salamander robot to propel itself forward in water. The lateral displacements are similar to those of the salamander with points of minimal displacement traveling from head to tail (arrows, Fig. 4A and B). The envelope of maximal lateral displacement has a more complex profile than that of the real salamander in which the maximal lateral displacement increases more or less monotonically from head to tail (Fig. 4C). In the robot, there is a bump in the envelope just above the hindlimb girdle. This is probably because the lack of a hinge joint at the girdle and the increased mass of the hindlimb module affect lateral displacements. Consistent with salamander kinematics and EMG recordings, an undulation wavelength of one body-length is maintained even when the frequency of oscillations is modified with the drive. The swimming velocities range from 0.07 to $0.12 \mathrm{~m} / \mathrm{s}$ (0.08 to 0.14 body lengths $/ \mathrm{s})$. In relative terms, the robot swims considerably slower than $P$. waltlii (from 0.4 to 1.2 body lengths/s). The difference likely results from a combination of three factors: The robot has lower frequencies, fewer actuated joints, and a less profiled body than $P$. waltlii. Nonetheless, considering the relatively simple design of the robot, its overall performance captures many elements of the salamander's locomotor behavior.

Lateral turning can be induced during both walking and swimming by applying asymmetrical drives between left and right sides of the body CPG. Such a mechanism is in agreement with the activity patterns of reticulospinal neurons observed during lateral turns in the swimming lamprey. See (23) and movie S2.

The model leads to the following four predictions. (i) It predicts that limb oscillators saturate at lower frequencies than body oscillators (hypothesis 3). The saturation could be due either to a spinal mechanism (i.e., limb oscillators are intrinsically limited to lower frequencies) and/or to a mechanism in the reticulospinal neurons (i.e., these neurons could stop transmitting the locomotor command to the limb oscillators if the signal exceeds a threshold). (ii) Hypothesis 4 predicts that motoneuron signals to limb and axial muscles should exhibit different oscillation frequencies for the same drive when body oscillators are isolated from limb oscillators. Experiments show this prediction to be true, see Section 4 of the Materials and methods. (iii) We predict that, similar to the lamprey, asymmetrical stimulation of the brain stem will lead to turning in salamanders. (iv) We predict that lesioning the neural pathways from limb centers to body centers will modify the walking gait — the body will tend to make traveling waves, and there will be a loss of coordination between limb movements and body undulations - but not the swimming mode.

The main implication of this study for vertebrate locomotion is to show how a tetrapod locomotion controller can be built on top of a primitive swimming circuit and explain the mechanisms of gait transition, the switch between traveling and standing waves of body undulations, and the coordination between body and limbs. This work extends models of gait transitions as bifurcation phenomena (25-27) by taking evolutionary modifications into account and proposing that the addition of oscillatory centers together with the modification of intrinsic and saturation frequencies in spinal oscillators could provide a general mechanism for the generation of multiple gaits in vertebrates.

Finally, this work also contributes to robotics. There is currently no well established methodology for controlling the locomotion of robots with multiple degrees of freedom, in particular for non-steady state locomotion in complex environments. CPGs offer an interesting approach to solving the problem of online trajectory generation by using the limit cycle behavior of coupled oscillators to produce the motor commands in real time. CPG-based control allows one to reduce the dimensionality of the locomotion control problem while remaining highly flexible to continuously adjust velocity, direction and type of gait according to the environmental context.

\section{References and Notes}

1. A. H. Cohen, in Neural Control of Rhythmic Movements in Vertebrates A. H. Cohen, S. Rossignol, S. Grillner, Eds. (Jon Wiley \& Sons, 1988) pp. 129-166.

2. K.-Q. Gao, N. H. Shubin, Nature 410, 574 (2001).

3. L. M. Frolich, A. A. Biewener, Journal of Experimental Biology 62, 107 (1992). 
4. M. A. Ashley-Ross, B. F. Bechtel, Journal of Experimental Biology 207, 461 (2004).

5. I. Delvolvé, T. Bem, J.-M. Cabelguen, Journal of Neurophysiology 78, 638 (1997).

6. P. Roos, Proc. ned. Akad. Wetten. C. 67, 223 (1964).

7. I. Delvolvé, P. Branchereau, R. Dubuc, J.M. Cabelguen, Journal of Neurophysiology 82, 1074 (1999).

8. M. Wheatley, K. Jovanovic, R. B. Stein, V. Lawson, Journal of Neurophysiology 71, 2025 (1994).

9. A. H. Cohen, P. Wallen, Exp. Brain Res. 41, 11 (1980).

10. S. Grillner et al., Trends in Neuroscience 18, 270 (1995).

11. M. J. Tunstall, A. Roberts, Journal of Physiology-London 474, 393 (Feb 1, 1994).

12. D. Combes, S. D. Merrywest, J. Simmers, K. T. Sillar, Journal of Physiology-London 559, 17 (Aug 15, 2004).

13. J. Cheng et al., The Journal of Neuroscience 18, 4295 (1998).

14. G. Székely, G. Czéh, "Organization of locomotion,” Frog Neurobiology, a Handbook 1976, pp. 765-792.
15. J. M. Cabelguen, C. Bourcier-Lucas, J. Dubuc, Journal of Neuroscience 23, 2434 (Mar 15, 2003).

16. S. Grillner, A. P. Georgopoulos, L. M. Jordan, in Neurons, networks and motor behavior P. S. G. Stein, S. Grillner, A. Selverston, D. G. Stuart, Eds. (MIT Press, Cambridge, MA, 1997).

17. B. Ermentrout, N. Kopell, SIAM Journal of Applied Mathematics 54, 478 (1994).

18. A. J. Ijspeert, Biological Cybernetics 84, 331 (2001).

19. T. Bem, J.-M. Cabelguen, O. Ekeberg, S. Grillner, Biological Cybernetics 88, 79 (2003).

20. A. J. Ijspeert, A. Crespi, J. M. Cabelguen, NeuroInformatics 3, 171 (2005).

21. T. L. Williams, K. A. Sigvardt, N. Kopell, G. B. Ermentrout, M. P. Rempler, J. of Neurophysiology 64, 862 (September, 1990).

22. S. Grillner et al., Brain Research Reviews 26, 184 (May, 1998).

23. Supporting online movies and text are available on Science Online.

24. B. Webb, Nature 417, 359 (May, 2002).
25. J.J. Collins, S.A. Richmond, Biological Cybernetics 71, 375 (1994).

26. G. Schoner, J. A. S. Kelso, Science 239, 1513 (1988).

27. M. Golubitsky, I. Stewart, P.-L. Buono, J. J. Collins, Nature 401, 693 (1999).

28. We acknowledge support from the Swiss National Science Foundation and the French "Ministère de la Recherche et de la Technologie" (ACI NIC 032362). We are grateful to Barbara Webb, Monica Daley, Bard Ermentrout, Alan Roberts, Gwendal Le Masson, and Stefan Schaal for useful comments, André Guignard and André Badertscher for their help in the construction of the robot, Isabelle Delvolvé for kinematic recordings, and Francesco Mondada for providing the PD motor controller.

\section{Supporting Online Material}

www.sciencemag.org

Materials and Methods

Movies S1.mov, S2.mov 